\title{
乌ु \\ Reversible State Transfer between Light and a Single Trapped Atom
}

\author{
A. D. Boozer, A. Boca, R. Miller, T. E. Northup, and H. J. Kimble \\ Norman Bridge Laboratory of Physics 12-33, California Institute of Technology, Pasadena, California 91125, USA
}

(Received 25 February 2007; published 8 May 2007)

\begin{abstract}
We demonstrate the reversible mapping of a coherent state of light with a mean photon number $\bar{n} \simeq 1.1$ to and from the hyperfine states of an atom trapped within the mode of a high-finesse optical cavity. The coherence of the basic processes is verified by mapping the atomic state back onto a field state in a way that depends on the phase of the original coherent state. Our experiment represents an important step toward the realization of cavity QED-based quantum networks, wherein coherent transfer of quantum states enables the distribution of quantum information across the network.
\end{abstract}

DOI: 10.1103/PhysRevLett.98.193601

PACS numbers: 42.50.Pq, 03.67.- a, 32.80.Pj

An important goal in quantum information science is the realization of quantum networks for the distribution and processing of quantum information [1], including for quantum computation, communication, and metrology [2-5]. In the initial proposal for the implementation of quantum networks [6], atomic internal states with long coherence times serve as "stationary" qubits, stored and locally manipulated at the nodes of the network. Quantum channels between different nodes are provided by optical fibers, which transport photons ("flying" qubits) over long distances [7]. A crucial requirement for such network protocols is the reversible mapping of quantum states between light and matter. Cavity quantum electrodynamics (QED) provides a promising avenue for achieving this capability by using strong coupling for the interaction of single atoms and photons [8].

Within this setting, reversible emission and absorption of one photon can be achieved by way of a dark-state process involving an atom and the field of a high-finesse optical cavity. For classical fields, this adiabatic passage process was first considered 20 years ago $[9,10]$, before being adapted to quantum fields [11] and specifically to the coherent transfer of quantum states between remote locations [6], with many extensions since then [12]. The basic scheme, illustrated in Fig. 1, involves a three-level atom with ground states $|a\rangle$ and $|b\rangle$ and excited state $|e\rangle$. An optical cavity is coherently coupled to the atom on the $b \leftrightarrow$ $e$ transition with rate $g$, and a classical field $\Omega(t)$ drives the atom on the $a \leftrightarrow e$ transition. If the $\Omega$ field is ramped adiabatically off $\rightarrow$ on, then state $|a, n\rangle$ evolves into $|b, n+1\rangle$, and state $|b, n\rangle$ remains unchanged, where $|a, n\rangle,|b, n\rangle$ denotes a state in which the atom is in ground state $a, b$ and there are $n$ photons in the cavity. Ramping $\Omega$ on $\rightarrow$ off implements the reverse transformation.

This process can be used to generate single photons by preparing the atom in $|a\rangle$ and ramping $\Omega$ off $\rightarrow$ on, thereby effecting the transfer $|a, 0\rangle \rightarrow|b, 1\rangle$ with the coherent emission of a single-photon pulse from the cavity $[6,11,13]$. Essential aspects of this process have been confirmed in several experiments [14-16], including tailoring of the single-photon pulse shape [15].
A distinguishing aspect of this protocol is that it should be reversible [6], so that a photon emitted from one system $A$ can be efficiently transferred to another system $B$. Furthermore, it should be possible to map coherent superpositions reversibly between the atom and the field:

$$
\left(c_{0}|b\rangle+c_{1}|a\rangle\right) \otimes|0\rangle \leftrightarrow|b\rangle \otimes\left(c_{0}|0\rangle+c_{1}|1\rangle\right) .
$$

Over the past decade, single photons have been generated in diverse physical systems [17]; however, most such sources are not in principle reversible, and for those that are, no experiment has verified the reversibility of either the emission or the absorption process.

In this Letter, we report an important advance related to the interface of light and matter by explicitly demonstrating the reversible mapping of a coherent optical field to and from the hyperfine ground states of a single, trapped Cesium atom [18]. Specifically, we map an incident coherent state with $\bar{n}=1.1$ photons into a coherent superposition of $F=3$ and $F=4$ ground states with transfer efficiency $\zeta=0.057$ [19]. We then map the stored atomic state back to a field state. The coherence of the overall process is confirmed by observations of interference between the final field state and a reference field that is phase coherent with the original coherent state, resulting in a fringe visibility $v_{a}=0.46 \pm 0.03$ for the adiabatic absorption and emission processes. We thereby provide the first verification of the fundamental primitive upon which the protocol in Ref. [6] is based.

As shown schematically in Fig. 2(a), our system consists of one Cs atom coupled to a high-finesse Fabry-Perot

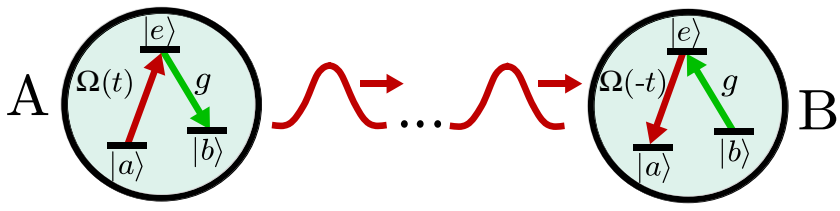

FIG. 1 (color online). Illustration of the protocol of Ref. [6] for quantum state transfer and entanglement distribution from system $A$ to system $B$. By expanding to a larger set of interconnected cavities, complex quantum networks can be realized. 


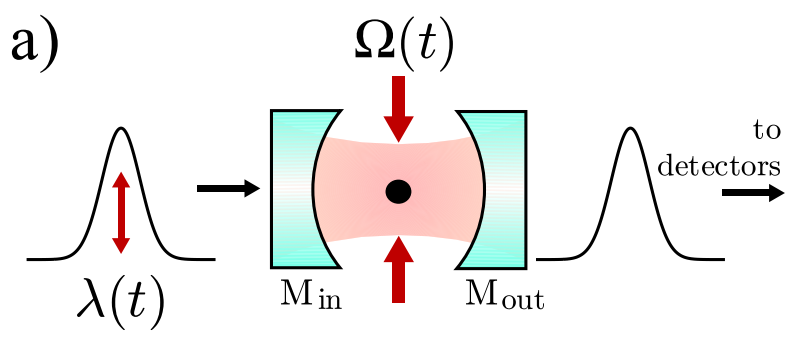

b)

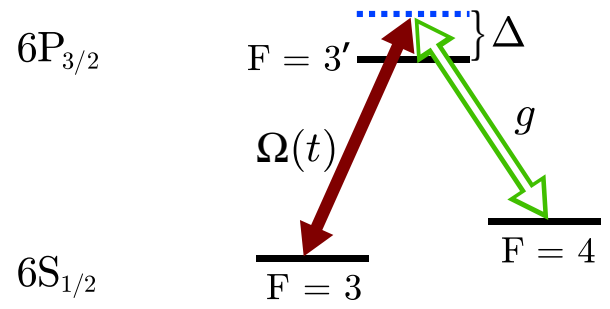

FIG. 2 (color online). (a) Schematic of the experiment. The probe $\lambda(t)$ resonantly drives the cavity through input mirror $M_{\mathrm{in}}$; the classical field $\Omega(t)$ excites the atom transverse to the cavity axis. Photons emitted from the output mirror $M_{\text {out }}$ are directed to a pair of avalanche photodiodes. (b) Atomic level diagram. Double arrow $g$ indicates the coherent atom-cavity coupling, and $\Omega(t)$ is the classical field. The cavity and $\Omega$ field are bluedetuned from atomic resonance by $\Delta$.

cavity. The cavity length is tuned so that a $\mathrm{TEM}_{00}$ mode is near resonance with the $6 S_{1 / 2}, F=4 \rightarrow 6 P_{3 / 2}, F=3^{\prime}$ transition of $\mathrm{Cs}$ at $852.4 \mathrm{~nm}$. The maximum atom-cavity coupling rate is $g_{0} / 2 \pi=16 \mathrm{MHz}$, while the cavity field and the atomic excited state decay at rates $(\kappa, \gamma) / 2 \pi=$ $(3.8,2.6) \mathrm{MHz} \ll g_{0}$. Thus, the system is in the strong coupling regime of cavity QED [8].

Atoms are dropped from a magneto-optical trap into the cavity and cooled into a far off-resonant trap (FORT) by a blue-detuned optical lattice (see $[8,20])$. The FORT excites another $\mathrm{TEM}_{00}$ cavity mode at the "magic" wavelength $935.6 \mathrm{~nm}$, creating nearly equal trapping potentials for all states in the $6 S_{1 / 2}, 6 P_{3 / 2}$ manifolds [21].

An atomic level diagram is shown in Fig. 2(b); the states used in the current scheme are ground $F=3,4$ and excited $F=3^{\prime}$ manifolds, corresponding to $|a\rangle,|b\rangle,|e\rangle$ in Fig. 1. The cavity is tuned to frequency $\omega_{C}=\omega_{4-3^{\prime}}+\Delta$, where $\omega_{4-3^{\prime}}$ is the frequency of the $4-3^{\prime}$ transition, and $\Delta / 2 \pi=10 \mathrm{MHz}$ is the cavity-atom detuning. A linearly polarized probe beam [22] drives the cavity at frequency $\omega_{C}$ with pumping strength $\lambda(t)$. An optical lattice drives the atom transverse to the cavity axis at frequency $\omega_{A}=$ $\omega_{3-3^{\prime}}+\Delta$ to provide a classical field with Rabi frequency $\Omega(t)$ [14]. The laser source for the optical lattice is phase locked in Raman resonance with the probe laser, so their relative detuning $\delta=\omega_{A}-\omega_{C}$ is phase stable and equal to the ground-state hyperfine splitting $\Delta_{\mathrm{HF}}=\omega_{3-3^{\prime}}-$ $\omega_{4-3^{\prime}}=(2 \pi)(9.193 \mathrm{GHz})$.

Our experimental procedure is as follows: after loading an atom into the FORT, we subject it to 2000 trials lasting a total of $360 \mathrm{~ms}$, where each trial consists of a series of discrete measurements performed on the atom. These measurements are used to quantify the coherence of the absorption process, as well as for calibrations and background monitoring. After these trials, we check that the atom has survived in the trap by attempting to generate 10000 single photons, which are detected by monitoring the cavity output with two single-photon counting avalanche photodiodes. We keep only the data from atoms that have survived all the trials. For most of the data that we keep, only a single atom is present in the trap, but occasionally two or more atoms may be loaded. From measurements performed during the 2000 trials, we determine that at least $80 \%$ of the data presented here involve a single atom.

For each trial, we prepare the atom in $F=4$ and then drive the system with a series of light pulses, as shown in Fig. 3. The classical field $\Omega(t)$ generates pulses $\Omega_{1,2}$, and the cavity probe $\lambda(t)$ generates pulses $\lambda_{1,2}$. For any given measurement within a trial, some of these pulses are on and the others are off. Pulse $\lambda_{1}$ is the coherent state that is to be mapped into the atom. The strength of this pulse is set so that there are $\bar{n}=1.1$ mode-matched photons at the face of the input mirror $M_{\mathrm{in}}$. Because of mirror losses [23], if no atom were present, this would give rise to a pulse inside the cavity with $\bar{n}=0.68$ photons. The falling edge of pulse $\Omega_{1}$ is used to perform the adiabatic absorption of $\lambda_{1}$. The intensity of the lattice light is such that when $\Omega_{1}$ is fully on, its Rabi frequency is $\sim 8 \gamma$, a value found to maximize the adiabatic absorption probability. When $\lambda_{1}$ is absorbed, some of the atomic population is transferred from $F=4$ to $F=3$. With $\lambda_{2}$ off, $\Omega_{2}$ allows us to determine the fraction of the population that has been transferred: if the atom is in $F=4$, then $\Omega_{2}$ does nothing, while if the atom is in $F=3$, then the rising edge of $\Omega_{2}$ transfers it back to $F=4$ and generates a single photon. Finally, with both pulses $\Omega_{2}$ and $\lambda_{2}$ on, we verify that $\lambda_{1}$ was absorbed coherently. The $\Omega_{2}$,

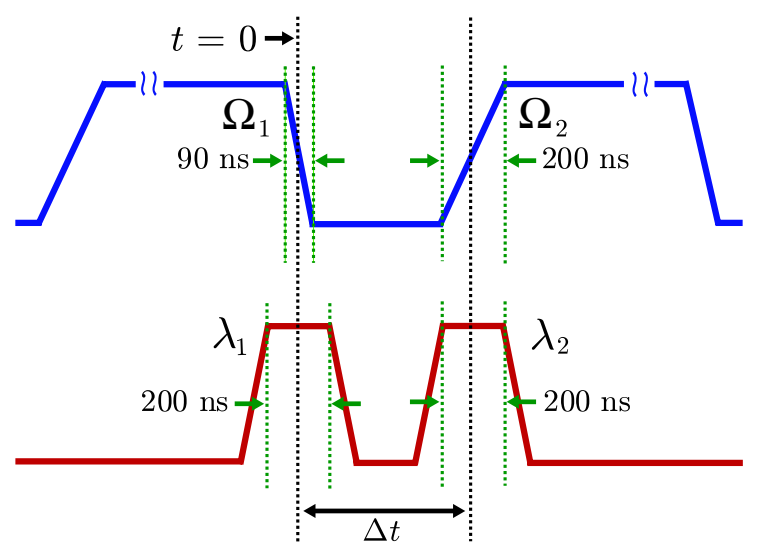

FIG. 3 (color online). Timing diagram: the upper curve shows the $\Omega_{1}$ and $\Omega_{2}$ pulses; the lower curve shows the $\lambda_{1}$ and $\lambda_{2}$ pulses. Each of these pulses can be turned on or off independently. Here $\Delta t$ is the delay between the falling edge of $\Omega_{1}$ and the rising edge of $\Omega_{2}$. By enabling various combinations of these pulses, and/or varying the relative phase $\theta$ between $\lambda_{1}$ and $\lambda_{2}$, we perform different measurements on the atom [28]. 
$\lambda_{2}$ pulses act together to generate a field inside the cavity; if $\lambda_{1}$ was absorbed coherently, then the amplitude of this field will depend on the relative phase $\theta$ between $\lambda_{1}, \lambda_{2}$.

This dependence can be understood by considering a simple model in which $\Omega_{2}$ and $\lambda_{2}$ act independently. With $\lambda_{2}$ off and $\Omega_{2}$ on, the $\Omega_{2}$ pulse transfers the atom from a superposition of $F=3,4$ into $F=4$ by generating a field $\alpha$ in the cavity whose phase depends on the phase of the atomic superposition. In turn, the phase of the original atomic superposition is set by the phase of $\lambda_{1}$. With $\lambda_{2}$ on and $\Omega_{2}$ off, the $\lambda_{2}$ pulse generates a field $\beta$ inside the cavity whose phase is set by $\lambda_{2}$. If $\Omega_{2}$ and $\lambda_{2}$ acted independently, then when both $\Omega_{2}$ and $\lambda_{2}$ were on, the fields $\alpha$ and $\beta$ would combine to give a total field $\alpha+\beta$, whose amplitude depends on the phase difference $\theta$ between $\lambda_{1}$ and $\lambda_{2}$. Because $\Omega_{2}$ and $\lambda_{2}$ do not act independently, this model is only approximately correct. Nevertheless, the phase of the final field still depends on $\theta$ for the coherent processes associated with $\lambda_{1,2}, \Omega_{1,2}$.

We first consider a series of measurements which demonstrate that the $\lambda_{1}$ pulse transfers more population from $F=4$ to $F=3$ in the presence of the $\Omega_{1}$ pulse than in its absence. We start with the atom in $F=4$ and apply the $\lambda_{1}$ pulse, either with the $\Omega_{1}$ pulse (adiabatic absorption, which consists of both coherent and incoherent components) or without it (only incoherent absorption $4 \rightarrow 3^{\prime}$, with spontaneous decay to $F=3$ ). In either case, $\lambda_{1}$ transfers some population from $F=4$ to $F=3$. To quantify the population transfer, we apply $\Omega_{2}$ and measure the probability that a single photon is detected within $1 \mu \mathrm{s}$ of the rising edge of $\Omega_{2}$ [24]. We thereby infer the fraction of the atomic population that was in $F=3$ [25]. For adiabatic absorption $\left(\Omega_{1}\right.$ on), we find that the probability $p_{a}$ for the atom to be transferred from $F=4$ to $F=3$ by $\lambda_{1}$ is $p_{a}=$

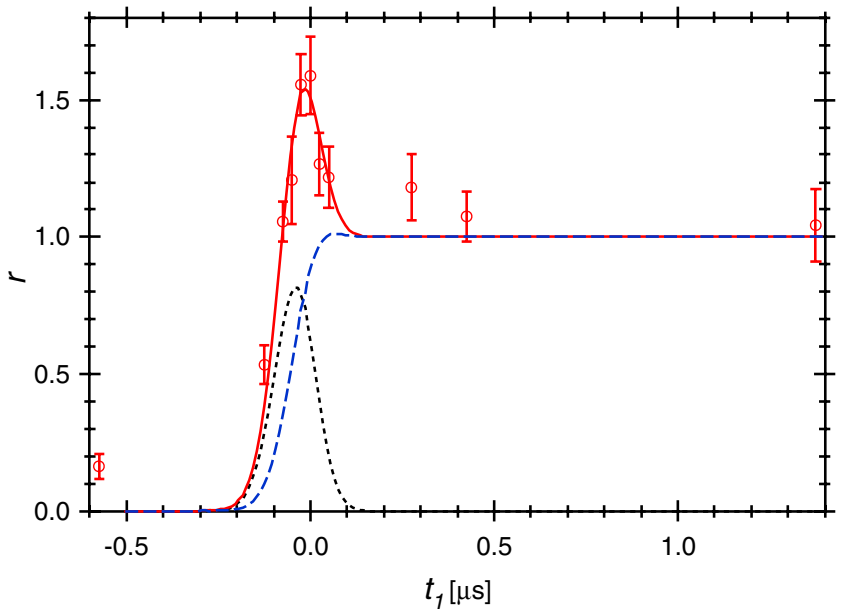

FIG. 4 (color online). Ratio $r$ of adiabatic transfer probability to incoherent transfer probability versus arrival time $t_{1}$ for the incident coherent pulse $\lambda_{1}$. Red data points $(\bigcirc): r$ versus $t_{1}$ (experiment). Solid red curve: $r$ vs $t_{1}$ (computer simulation). Dotted black curve: coherent component $r^{c}$ vs $t_{1}$ (simulation). Dashed blue curve: incoherent component $r^{i}$ vs $t_{1}$ (simulation).
$0.063 \pm 0.002$, whereas for incoherent absorption $\left(\Omega_{1}\right.$ off), the probability is $p_{i}=0.046 \pm 0.001$. The ratio of the adiabatic to the incoherent absorption probability is $r=p_{a} / p_{i}=1.38 \pm 0.04$.

As shown in Fig. 4, we vary the arrival time $t_{1}$ of the $\lambda_{1}$ pulse and study the effect on the adiabatic-to-incoherent ratio $r$ [26]. This ratio is maximized when $\lambda_{1}$ is well aligned with the falling edge of $\Omega_{1}$ at $t=0$. If $\lambda_{1}$ arrives too early $\left(t_{1} \ll 0\right)$, then any population that it transfers from $F=4$ to $F=3$ is pumped back to $F=4$ by $\Omega_{1}$. If $\lambda_{1}$ arrives too late $\left(t_{1} \gg 0\right)$, then $\Omega_{1}$ is already off, resulting in incoherent transfer with $r=1$.

Figure 4 also shows the results of a computer simulation of the absorption process. The simulation predicts values for $p_{a}$ and $p_{i}$ and therefore the ratio $r=p_{a} / p_{i}$. The correspondence between our simulation and the actual measurements of $r$ vs $t_{1}$ in Fig. 4 is qualitatively reasonable (the only free parameter in the simulation is the atomcavity coupling $g$, which we set to $g / g_{0}=0.44$ ). The simulation can also be used to partition $p_{a}$ into a coherent component $p_{a}^{c}$ and an incoherent component $p_{a}^{i}$. We define the coherent component of $r$ by $r^{c}=p_{a}^{c} / p_{i}$, the incoherent component of $r$ by $r^{i}=p_{a}^{i} / p_{i}$, and plot $r^{c}, r^{i}$ vs $t_{1}$ in Fig. 4. The simulation indicates that the value of $t_{1}$ for which the adiabatic absorption process is maximally coherent is roughly the value of $t_{1}$ that maximizes the adiabatic transfer probability, and suggests that for this value of $t_{1}$ the adiabatic absorption process has appreciable coherence, with $r^{c} / r^{i} \simeq 1$.

In Fig. 5, we present measurements that demonstrate that the adiabatic absorption process is indeed coherent. As before, we prepare the atom in $F=4$ and apply $\lambda_{1}$, either

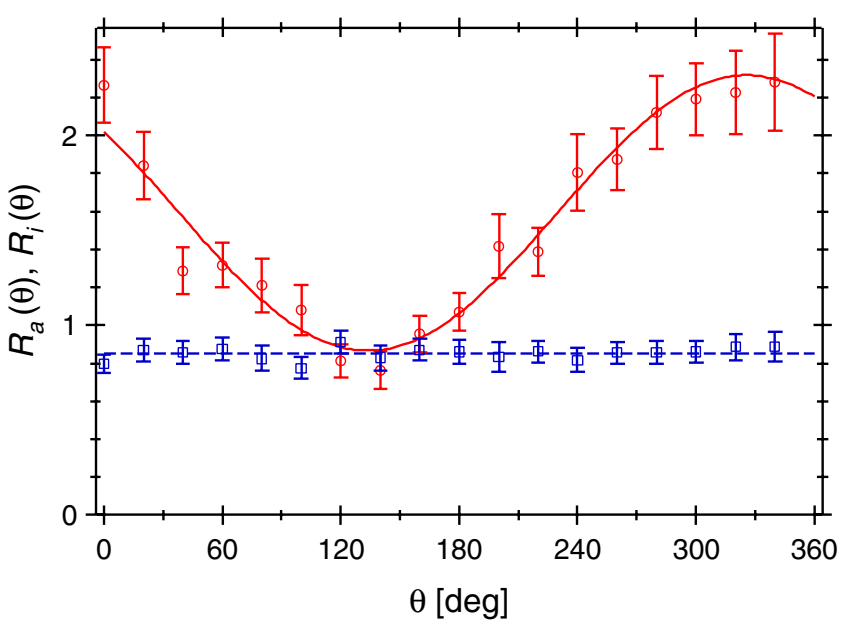

FIG. 5 (color online). Ratios $R_{a}(\theta), R_{i}(\theta)$ for photon generation as a function of the relative phase $\theta$ between the $\lambda_{1,2}$ fields. Red data points $(\bigcirc): R_{a}(\theta)$ for adiabatic state transfer with $\Omega_{1}$ on. Blue points $(\square): R_{i}(\theta)$ for the incoherent process with $\Omega_{1}$ off. The full curve is a fit to obtain the fringe visibility $v_{a} \simeq$ $0.46 \pm 0.03$. On average, each point represents about 130 atoms. The error bars represent statistical fluctuations from atom to atom. 
with or without $\Omega_{1}$, followed by $\Omega_{2}$. But now we add the $\lambda_{2}$ pulse, which overlaps with the rising edge of $\Omega_{2}$. If the $\lambda_{1}$ pulse is absorbed coherently, then the amplitude of the field generated by the combined action of $\Omega_{2}$ and $\lambda_{2}$ will depend on the relative phase $\theta$ of $\lambda_{1}$ and $\lambda_{2}$. By recording the cavity output from $M_{\text {out }}$ as a function of $\theta$ and observing this dependence, we can verify that the $\lambda_{1}$ pulse was absorbed coherently. To accomplish this, we repeat the above sequence for different values of $\theta$, where for each relative phase, we measure the mean number of photons $n(\theta)$ emitted from the cavity within a fixed detection window [24]. We take data both with $\Omega_{1}$ on and off, so as to obtain results $n_{a}(\theta)$ and $n_{i}(\theta)$ both for adiabatic and incoherent absorption. Figure 5 plots $R_{a}(\theta)=n_{a}(\theta) / n_{a}\left(\theta_{0}\right)$ and $R_{i}(\theta)=n_{i}(\theta) / n_{i}\left(\theta_{0}\right)$, where $\theta_{0}$ is a fixed phase. Note that these ratios, rather than the photon numbers themselves, are employed in order to cancel small, slow drifts in the intensity of the light beams. Significantly, we observe an appreciable phase dependence with visibility $v_{a}=$ $0.46 \pm 0.03$ for the adiabatic absorption curve $R_{a}(\theta)$, while no such variation is recorded for the incoherent absorption curve $R_{i}(\theta)$.

The fringe visibility is limited by the intrinsic incoherent component of the absorption process, as well as by the mismatch in amplitudes and pulse shapes for the $\alpha, \beta$ fields. For the results shown in Fig. 5, a 200 ns detection window is used around the peak of the emission process. If we increase the detection window to $1 \mu \mathrm{s}$, thus degrading the pulse shape overlap, the visibility drops to $v_{a}=$ $0.18 \pm 0.01$.

In conclusion, we have demonstrated the reversible transfer of a coherent pulse of light to and from the internal state of a single trapped atom, which represents a significant step toward the realization of quantum networks based upon interactions in cavity QED. Explicitly, we have presented a detailed investigation of the adiabatic absorption of an incident coherent state with $\bar{n}=1.1$ photons. A fraction $p_{a}=0.063$ of the atomic population has been transferred from $F=4$ to $F=3$, with the efficiency of the transfer being $\zeta \equiv p_{a} / \bar{n}=0.057$. Here $\zeta$ provides an estimate of the efficiency that could be obtained if we adiabatically absorbed a single-photon state instead of a coherent state, and should be compared to the much lower efficiencies possible in free space.

The factors that limit the transfer efficiency include the passive mirror losses [23], the fact that our cavity mirrors $M_{\text {in }}, M_{\text {out }}$ have equal transmission coefficients $T_{\text {in }}=T_{\text {out }}$ (as opposed to $T_{\text {in }} \gg T_{\text {out }}$ for a single-sided cavity), and the coupling of the atom to both polarization modes of the cavity. Even in the ideal case without scatter and absorption losses in the mirrors, for a three-level atom coupled to a two-sided cavity ( $T_{\text {in }}=T_{\text {out }}$ ) with two modes, the maximum possible adiabatic transfer probability would be $\zeta=$ 0.25 . By implementing a single-sided cavity with losses as achieved in Ref. [27], we estimate that $\zeta$ could be im- proved to $\zeta \sim 0.9$ for coupling schemes with a single polarization.

This research is supported by the National Science Foundation and the Disruptive Technology Office.

[1] P. Zoller et al., Eur. Phys. J. D 36, 203 (2005).

[2] L.-M. Duan and H. J. Kimble, Phys. Rev. Lett. 92, 127902 (2004).

[3] C. H. Bennett, Phys. Today 48, No. 10, 24 (1995).

[4] A. Ekert, Phys. Rev. Lett. 67, 661 (1991).

[5] G. Giovannetti et al., Science 306, 1330 (2004).

[6] J. I. Cirac et al., Phys. Rev. Lett. 78, 3221 (1997).

[7] H.-J. Briegel et al., Phys. Rev. Lett. 81, 5932 (1998).

[8] R. Miller et al., J. Phys. B 38, S551 (2005).

[9] J. Oreg et al., Phys. Rev. A 29, 690 (1984).

[10] J. R. Kuklinski et al., Phys. Rev. A 40, 6741 (1989).

[11] A. S. Parkins et al., Phys. Rev. Lett. 71, 3095 (1993).

[12] N. V. Vitanov et al., Adv. At. Mol. Opt. Phys. 46, 55 (2001).

[13] L.-M. Duan et al., Phys. Rev. A 67, 032305 (2003).

[14] J. McKeever et al., Science 303, 1992 (2004).

[15] M. Keller et al., Nature (London) 431, 1075 (2004).

[16] M. Hijlkema et al., quant-ph/0702034.

[17] Special issue on Focus on Single Photons on Demand, edited by P. Grangier et al. [New J. Phys. 6, 85 (2004)].

[18] This mapping could also be demonstrated by absorbing a single-photon state, but we use a coherent state instead, because its phase information allows us to verify explicitly the reversibility of the absorption process.

[19] Since we use a coherent state rather than a coherent superposition of $n=0,1$ Fock states, Eq. (1) only approximately describes our system. For a $\bar{n}=0.68$ coherent state (i.e., $\bar{n}=1.1$ at the face of $M_{\text {in }}$ ), the fraction of the population in the $n=0,1$ subspace is $\simeq 0.85$.

[20] A. D. Boozer et al., Phys. Rev. Lett. 97, 083602 (2006).

[21] J. McKeever et al., Phys. Rev. Lett. 90, 133602 (2003).

[22] Our cavity supports two nearly degenerate modes with orthogonal linear polarizations along the $\hat{x}$ and $\hat{y}$ axes, where $\hat{z}$ denotes the cavity axis. The cavity probe drives either the $\hat{x}$ or the $\hat{y}$ mode; a polarizer at the cavity output allows only the $\hat{x}$ component to reach the detectors.

[23] C. J. Hood et al., Phys. Rev. A 64, 033804 (2001).

[24] For these measurements, $\Delta t=290 \mathrm{~ns}$. The probe is polarized along $\hat{y}$; for the case when $\lambda_{2}$ is on, this ensures that the emerging signal is not dominated by the component of $\lambda_{2}$ that is transmitted by the cavity.

[25] This involves subtracting a background probability of 0.0025 , which we determine by pumping the atom to $F=$ 4 and applying $\Omega_{2}$, and dividing by the single photon generation efficiency of 0.036 , which we determine by pumping the atom into $F=3$ and applying $\Omega_{2}$.

[26] For these measurements, $\Delta t=2 \mu \mathrm{s}$, and the probe is polarized along $\hat{x}$.

[27] G. Rempe et al., Opt. Lett. 17, 363 (1992).

[28] The phase $\theta$ is set by the phase difference of rf pulses driving an acousto-optic modulator in the probe beam. 